\title{
Etude De La Variabilite Structurale Et Floristique Des Forets Sur Terre Ferme En Chefferie De Bahema-Boga (Province De L'ituri, RDC)
}

\author{
Benoit Mposo Nsangua \\ Département d'écologie et Gestion des Ressources Végétales, \\ Université de Bunia (RDC) \\ Prof. Hippollyte Nshimba Nseya \\ Prof. Faustin Boyemba B. \\ Prof. Roger Katusi L. \\ Prof. Faustin Mbayo M.
}

Faculté de Gestion des Ressources Renouvelables,

Université de Kisangani (RDC)

Laurianne Mbuyu M. (Attachée de Recherche)

Centre de Recherche Multidisciplinaire Pour le Développement (CRMD)-

Bunia, Section de Sociologie, Province de l'Ituri (RDC)

Doi: 10.19044/esj.2018.v14n30p500 URL:http://dx.doi.org/10.19044/esj.2018.v14n30p500

\begin{abstract}
The knowledge of the structural and floristic variability of forest is the principal tool which makes it possible to know the floristic composition of forest in an area. This paper focuses on analyzing the structural and floristic variability between compounds of forest on firm earth in chieftainship Bahema-boga in Ituri Province in Democratic Republic of Congo (DRC). The study provides knowledge and education to those responsible for environmental protection and those who are concerned with the forest based on the floristic potential for the conservation and the sustainable management of the forests. The structure and the floristic composition vary from one forest to another. The quantity of carbon stoke also vary due to the function of structures and floristic composition of forests. The forests play an important role in the reduction of $\mathrm{CO} 2$. The inventories of trees whose diameter is $\geq 10$ $\mathrm{cm}$ have allowed us to collect the data to analyze this structural and floristic variability in a sample of 3 ha (plots) of forests in firm earth. The results obtained indicate that 812 inventoried individuals (trees) are extended in 16 species, 11 kinds, and 8 famillies. The total earth surface of inventoried individuals is $32,06 \mathrm{~m}^{2} / \mathrm{h}$. The species of Cynometra sessiliflora dominated those forests with 408 individuals $(50,25 \%)$. The diametric class of the most
\end{abstract}


elevated inventoried plants are located between $10-20 \mathrm{~cm}(63,17 \%)$. The family of the Fabaceae is better represented in those forests $(73,65 \%)$.

Keywords: Variability, Structural, floristic, earth surface, Chieftainship, Bahema-Boga, Province, Ituri, Democratic Republic of Congo

\section{Resume}

La connaissance de la variabilité structurale et floristique des forêts est le principal outil qui permet de connaître la composition floristique des forêts d'une région donnée. L'objectif de cette étude est d'analyser la variabilité structurale et floristique entre les parcelles des forêts sur terre ferme en chefferie de Bahema-boga dans la province de l'Ituri en République Démocratique du Congo (RDC). Cette étude renseigne les responsables de la protection environnementale et ceux qui sont intéressés par les forêts sur le potentiel floristique pour la conservation et la gestion durable des forêts. La structure et la composition floristique varient d'une forêt à l'autre. La quantité des carbones stockée varie aussi en fonction des structures et de la composition floristique des forêts. Les forêts jouent un rôle primordial dans la réduction des émissions de $\mathrm{CO}_{2}$. Les inventaires des arbres dont le diamètre est $\geq 10 \mathrm{~cm}$ nous ont permis de collecter les données pour analyser cette variabilité structurale et floristique dans un échantillon de 3 ha (Parcelles) aléatoires dans les forêts sur terre ferme. Les résultats obtenus indiquent que 812 individus inventoriés (arbres) sont répartis dans 16 espèces, 11 genres et 8 familles. La moyenne de la surface terrière totale des individus inventoriés est de $32.06 \mathrm{~m}^{2} /$ ha. L'espèce de Cynometra sessiliflora domine ces forêts avec 408 individus $(50,25 \%)$. La classe diamétrique des plantes inventoriées la plus élevée se situe entre $10-20 \mathrm{~cm}$, soit 513 individus (63,17\%). La famille des Fabaceae est la mieux représentée et diversifiée dans ces forêts $(73,65 \%)$.

Mots clés: Variabilité, structurale, floristique, surface terrière, Chefferie, Bahema-Boga, Province, ituri, République démocratique du Congo

\section{Introduction}

La République Démocratique du Congo (RDC) compte une superficie de $1.280 .042 .16 \mathrm{~km}^{2}$; les formations essentiellement forestières couvrent environ 54,6\% de sa superficie. La forêt dense humide vient au premier rang avec $68.14 \%$ de couverture, suivie des forêts denses sèches dégradées (12 $\%$ ), des forêts secondaires $(9,5 \%)$, des forêts sur sol hydromorphe $(6,92 \%)$, des forêts de montagne $(3,14 \%)$, les galeries forestières et la forêt de mangrove sont moins représentées avec respectivement 0,19 et $0,04 \%$ de la superficie forestière (Ministère de l'environnement et de la conservation de la 
nature de la RDC, 1997). Le problème des forêts est généralement lié à la méconnaissance de leur contenu et à une maîtrise insuffisante de divers mécanismes écologiques s'établissant entre les espèces et leur environnement (Sonké, 1998). Les forêts tropicales localisées à cheval de l'équateur, entre les tropiques, présentent les variabilités structurales et floristiques selon les différentes zones physiographiques (Senterre, 2005).

La connaissance de la variabilité structurale et floristique des forêts constitue un outil de base pour connaitre la composition floristique des forêts d'une région donnée car elle renseigne sur le potentiel floristique présent dans la région pour la conservation et la gestion durable des forêts (Pélissier et al., 2010). La quantité des carbones stockée dans les plantes varie en fonction de la structure et la composition floristique des forêts. De la même manière les forêts tropicales présentent de manière qualitative et quantitative des différences dans la composition floristique et la structure forestière d'une région à une autre, étant évident que les raisons internes de ces individualisations se présentent non seulement dans l'ensemble du massif forestier tropical mais également à l'intérieur de chaque cycle forestier. C'est dans cette optique que nous avons mené une étude pour analyser la variabilité structurale et floristique des forêts sur terre ferme en chefferie de BahemaBoga dans la province de l'Ituri à l'Est de la RDC. Or, en dépits des recherches réalisées dans les forêts du bassin du Congo (RDC), la présente étude constitue donc une réponse positive à l'insuffisance des recherches scientifiques en écologie forestière dans la région du Bassin du Nil, particulièrement en chefferie de Bahema-Boga, prouvée par le manque d'aménagement des forêts dans cette région de l'Est de la République Démocratique du Congo.

\section{Matériel et Méthodes}

\section{Présentation du Milieu d'étude}

La chefferie de Bahema-Boga est située en Province de l'Ituri, République Démocratique du Congo, à cheval sur la partie Nord de la Province du Nord- Kivu et la partie Sud de la Province de l'Ituri en territoire d'Irumu, entre $29^{\circ} 56^{\prime}$ et $30^{\circ} 15^{\prime}$ des longitudes Est et $00^{\circ} 29^{\prime}$ et $01^{\circ} 06^{\prime}$ des latitudes Nord. Il couvre environ $3200 \mathrm{~km} 2$ au Nord-est du pays, au Sud du lac Albert. La limite de la chefferie est formée par la rivière Semliki qui délimite également la frontière avec l'Ouganda.

La région d'étude présente des forêts diversifiées évoluant de la rivière Semliki vers l'intérieur de la RDC en dessinant un relief relativement marqué de $600 \mathrm{~m}$ à $1400 \mathrm{~m}$ au centre. La plaine alluviale parfois inondée (la vaste dépression du rift Albertin), laisse place à des zones vallonnées boisées, des zones de plateaux et d'escarpements rocheux qui caractérisent la zone. Les sols sont variés ; argileux, sablonnés et argilo sablonnés offrant des possibilités de pratiquer des cultures diversifiées. Le climat tropical humide de la zone 
d'étude entraîne des précipitations importantes comprises entre 1.200 et $1.700 \mathrm{~mm}$ par an, valeur enregistrée au cours de la saison des pluies (Cléments, 1916).

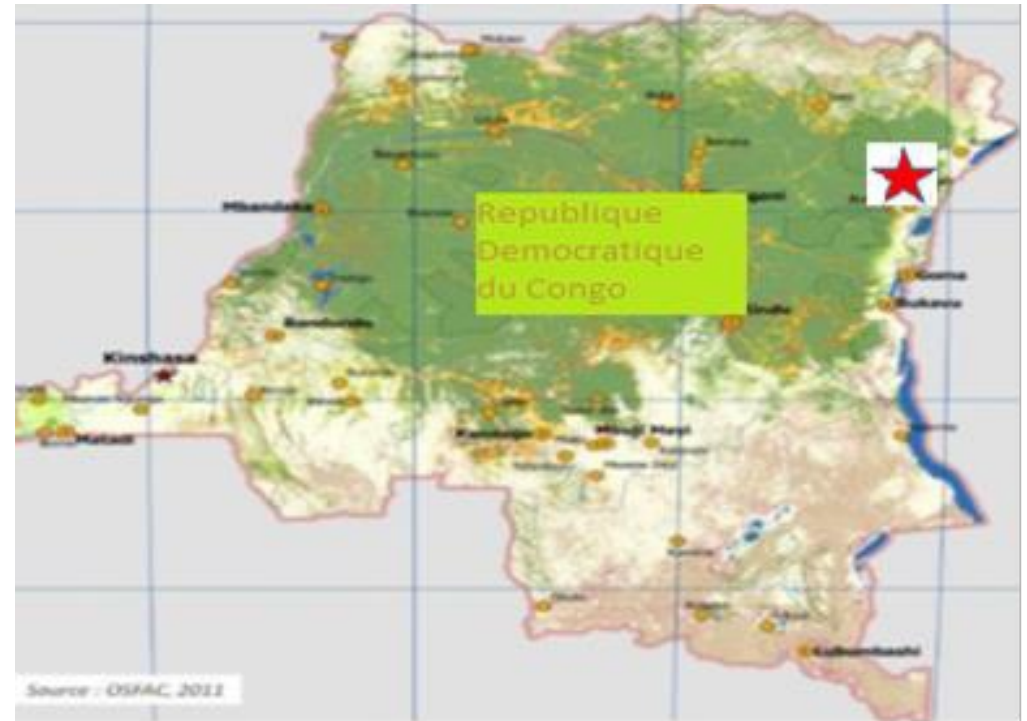

Figure 1. Carte 1 : Pertes de couvert forestier en RDC, 2000-2010 (source : Mecnt, 2010)

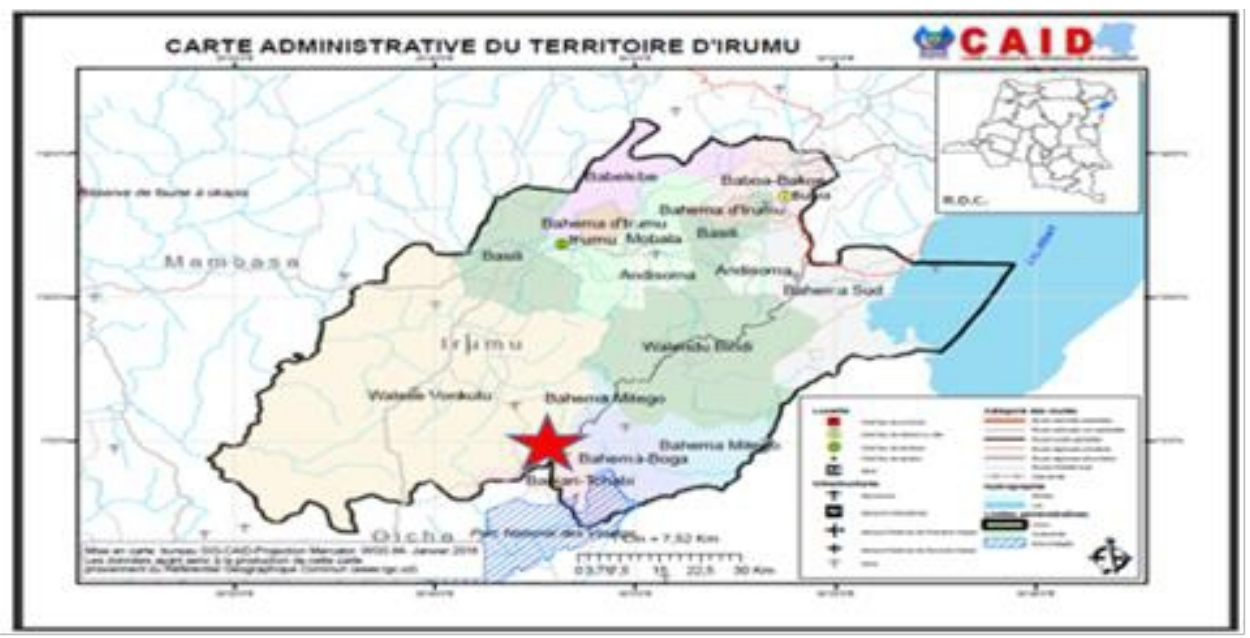

Figure 2. Carte administrative de la zone d'étude (Mise en carte: bureau SIG-CAIDProjection Mercator, WGS 84- Janvier 2016, www.rgc.cd)

= Lieu d'étude

\section{Matériel}

Pour prélever nos données, nous avons utilisé les matériels ci-après : l'appareil GPS marque GARMIN (650) pour prendre les coordonnées géographiques , l'appareil Photographique numérique pour la prise des photos 
utiles, un pentadécamètre nous a permis de délimiter la parcelle d'étude et de mesurer la distance de layon d'inventaire dans une parcelle, le mètre ruban de $50 \mathrm{~m}$ a permis à mesurer le diamètre et les circonférences des arbres inventoriés à la hauteur de la poitrine $(\mathrm{Dhp}) \geq 10 \mathrm{~cm}$, soit à $1,30 \mathrm{~m}$ au-dessus du sol des plantes dans les différentes parcelles d'inventaire, le sécateur a servi à couper les échantillons des plantes à identifier, le carnet pour noter les informations utiles, le marqueur pour marquer les arbres déjà identifiés et la machette pour l'ouverture des layons.

\section{Méthodes}

\section{Collecte des Données et Identification des Spécimens}

Pour atteindre nos objectifs, les inventaires des arbres nous ont permis de collecter les données pour analyser la variabilité structurale et floristique dans un échantillon de 3 ha (parcelles) aléatoires dans les forêts sur terre ferme. Cependant, dans ces parcelles, l'inventaire des individus uniquement au diamètre à la hauteur de poitrine $(\mathrm{Dhp}) \geq 10 \mathrm{~cm}$ a été effectué. La détermination des noms scientifiques et des familles de chaque espèce végétale inventoriée a été possible, soit directement sur le terrain ou soit à l'herbarium de la faculté des Sciences de l'Université de Kisangani. L'encodage standardisé des noms des taxons (espèces et familles) s'est fait suivant Lejoly et al. (2010) basé sur la classification « Angiosperm Phylogeny Group » (APG III). Un herbier des plantes type de la zone a été constitué et amené à l'herbarium de la Faculté des sciences de l'Université de Kisangani (RDC) pour identification par confrontation avec les spécimens existants.

\section{Dispositif d'échantillonnage}

Nous avons mis en place les parcelles dans les forêts, dont chacune des parcelles avait une superficie de 1 hectare, soit $100 \mathrm{~m} \times 100 \mathrm{~m}$ de côté. Ces parcelles étaient basées sur les protocoles de Dallmeier et al. (1992) et Picard et al. (2008).

- Le dispositif d'une parcelle d'étude dans les forêts est représenté par la figure ci-après. (Figure 3 ).

- Legende; $\longleftrightarrow$ = Layon

$\uparrow=$ Distance de $25 \mathrm{~m}$ d' inventaire entre deux layons

- - - - - = Milieu separant la zone d'inventaire entre deux layons $\rightarrow=$ Distance d'un côté de la parcelle 


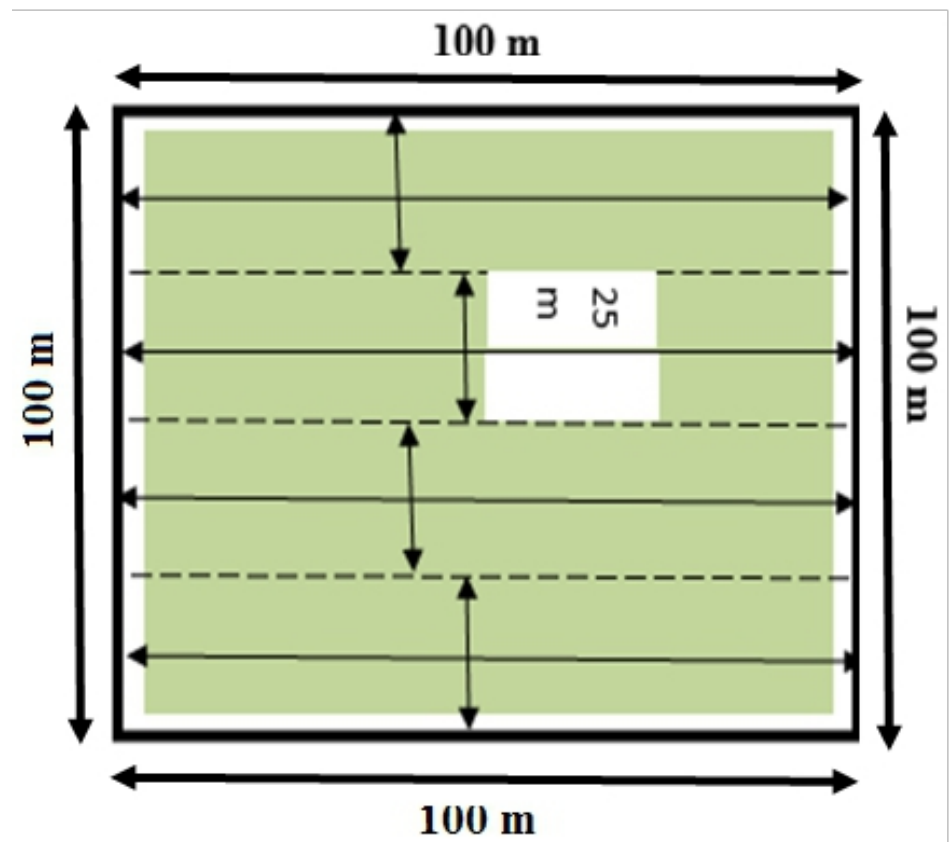

Figure 3. Représentation du dispositif d'échantillonnage d'une parcelle et au sein d'une parcelle d'étude

\section{Analyse Des Données}

L'appréciation de la variabilité structurale et floristique dans les parcelles d'étude a tenu compte de l'analyse de deux paramètres, notamment les paramètres structurales (la densité des arbres, la surface terrière, la dominance des taxons, Dominance relative, les indices de valeur d'importance des taxons, la structure diamétrique et les paramètres floristiques).

- La similarité floristique entre les parcelles d'étude est évaluée par l'indice de
$\left.\mathrm{CMH}=\frac{2 \Sigma(\mathrm{a}}{(\mathrm{da}+\mathrm{d}}\right)$
us d'un site $\mathrm{a} ;$
Morisita dont la formule est formule :
$a N$ indique le nombre d'individus d'un site $\mathrm{a}$;
$b N$ indique le nombre d'individus d'un site $\mathrm{b}$;
ani indique le nombre d'individus de l'espèce i dans le site $a$;
bni indique le nombre d'individus de l'espèce $\mathrm{i}$ dans le site $\mathrm{b}$;
$d a:$ nombre d'espèces spécifiques au site $\mathrm{a}$;
$d b$ indique le nombre d'espèces spécifiques au site b (Magurran, 2004).

- Le coefficient de Variation (CV) est utilisé pour apprécier l'ampleur de dispersion des arbres dans le milieu d'étude. Plus la valeur du CV est élevée, plus la dispersion des plantes est grande. 
Ce coefficient se calcule par la formule ; $C V=\frac{\delta \times 100}{M} \quad$ Où : CV indique le coefficient de variation, $M=$ moyenne et $\delta=$ écart type.

-Si CV > 30\%, la dispersion des arbres inventoriés est forte et leur distribution est hétérogène, $-\mathrm{Si} \mathrm{CV}<15 \%$, la dispersion des arbres inventoriés est faible et leur distribution est homogène et

-Si CV est entre $15 \%$ et $30 \%$, la dispersion des arbres inventoriés est plus ou moins faible et leur distribution est relativement homogène.

- La Diversité des taxons entre les parcelles étudiées a servi aussi à comparer la composition floristique des différents sites étudiés. Elle se calcule par ;

Div. Rel. $=\frac{\text { Nombre d'individus au sein d'une espece } / \text { famille }}{\text { nombre total d'espèces/famille }} \boldsymbol{x} 100$

- La Densité est utilisée pour connaître le nombre des individus recensés par unité de surface (Hectare). Elle se calcule en considérant le nombre d'individus d'une espèce ou d'une famille par rapport au nombre total des individus de ces taxons dans l'échantillon (Ibanda, 2012). Elle se calcule par l'équation :

$$
\text { Drsp }=\frac{\mathrm{ns}}{\mathrm{Ns}} \times 100
$$

Où : Drsp = densité des espèces, Ns = nombre total des espèces dans l'échantillon, $\mathrm{ns}=$ Nombre d'individus pour une espèce.

- La surface terrière sert à calculer la surface exprimée en m2 de la section transversale du tronc des arbres au niveau de la hauteur de la poitrine (dbh). Elle est calculée par l'équation : $S T=\frac{\pi D^{2}}{4}$ (Gounot, 1969) où $\pi=3,14$; ST = surface Terrière qui s'exprime en $\mathrm{m} 2$ par ha et $\mathrm{D}=$ Diamètre à la hauteur de la poitrine.

- L'analyse de la structure diamétrique nous renseigne avec précision sur le nombre des tiges inventoriées par classe de diamètre de $10 \mathrm{~cm}$ afin de comparer les structures diamétriques des différentes parcelles d'inventaire.

- Les indices des valeurs d'importance des taxons (Espèces et familles).

- Ces indices permettent d'identifier les entités dominantes d'une communauté ou d'un compartiment, c'est-à-dire, les espèces et les familles ayant une forte valeur écologique. Reitsma (1988) considère qu'une espèce ou une famille est écologiquement importante ou dominante lorsque IVI ou IFV $>10$.

Ils se calculent par les formules suivantes (Sabongo, 2015) : 
-IVI $=$ Densité relative + Dominance relative + Fréquence relative - FIV $=$ Densité relative + Dominance relative + Diversité relative

\section{Résultats}

\section{Densité et Surface terrière Densité des arbres}

Pour chaque parcelle (relevé) la surface terrière $\left(\mathrm{m}^{2} / \mathrm{ha}\right)$ et le diamètre $(\mathrm{cm})$ ont été calculés. Le nombre d'individus inventoriés, le nombre d'espèces et le nombre des genres et des familles par parcelle sont donnés dans le Tableau 1 ci-dessous. Une forte densité au sein des parcelles des forêts sur terre ferme est observée dans (P1) avec 384 individus inventoriés. La densité la plus faible est obtenue dans la parcelle (P3) avec 195 individus, ce qui montre une forte variabilité en comparant le nombre d'individus inventoriés au sein de chaque parcelle et entre les parcelles de ces forêts (ANOVA : Fstatistic $=0.03747 ;$ p-value $=0.8783$.

Légende: $P 1, P 2$ et $P 3=$ Parcelles d'inventaire, Nbre esp $=$ Nombre d'espèces, Nbre ind/ha. = Nombre d'individus inventoriés par hectare, Nbre.G $=$ Nombre des genres, Nbre Fam. $=$ Nombre de Familles et $S T=$ Surface terrière en $\mathrm{m}^{2} / \mathrm{ha}$.

Tableau 1. La densité et la surface terrière pour chaque parcelle étudiée

\begin{tabular}{lcccccc}
\hline Parcelles & N.ind/ha. & Nbre.Esp & Nbre.G. & Nbre..fam. & Dia. > 10 & ST \\
\hline P1 & 384 & 10 & 9 & 7 & 60,5 & 17,61 \\
\cline { 2 - 7 } P2 & 233 & 9 & 7 & 6 & 63,69 & 8,66 \\
P3 & 195 & 11 & 9 & 6 & 31,84 & 5,78 \\
Moyenne & 203 & 16,8 & 15,3 & 10,5 & 83,66 & 8 \\
Ecart type & 22,1 & 4,95 & 3,89 & 3,61 & 47,17 & 7,38 \\
CV (\%) & 10,9 & 29,6 & 25,5 & 0,34 & 56,38 & 92,25 \\
TOTAL & $\mathbf{8 1 2}$ & $\mathbf{1 6}$ & $\mathbf{1 1}$ & $\mathbf{8}$ & $\mathbf{6 3 , 6 9}$ & $\mathbf{3 2 , 0 6}$ \\
\hline
\end{tabular}

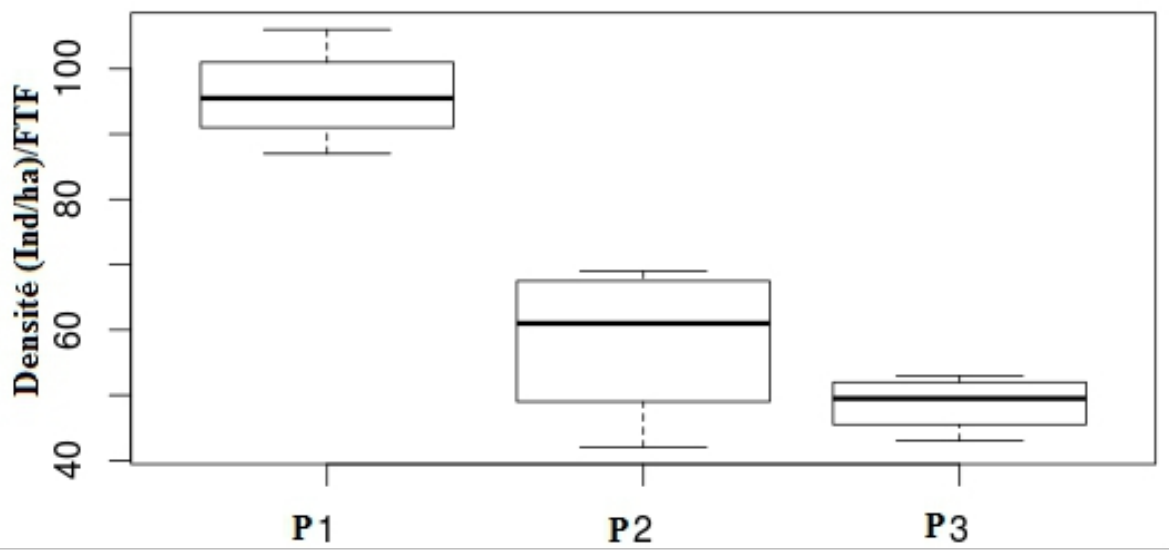

Figure 4. Boîte de représentation des densités au sein des parcelles des forêts sur terre ferme 
Ces boîtes représentent l'intervalle dans lequel sont groupés 50\% d'observations des densités au sein des parcelles des forêts sur terre ferme; la barre épaisse à l'intérieur de chaque boîte indique la densité moyenne, la barre basse indique la densité minimale et la barre haute indique la densité maximale.

\section{Surface Terrière}

La comparaison des surfaces terrières entre les parcelles de ces forêts (ANOVA : F-statistic $=0.6941 ; \quad$-value:= 0.5578) montre une forte variabilité remarquable des surfaces terrières des individus inventoriés dans les parcelles de ces forêts, où la parcelle (P1) présente une surface terrière la plus élevée $\left(17,61 \mathrm{~m}^{2} / \mathrm{ha}\right)$ tandis que la valeur la plus faible de la surface terrière est observée dans la parcelle $(\mathrm{P} 3)\left(5,78 \mathrm{~m}^{2} / \mathrm{ha}\right)$.

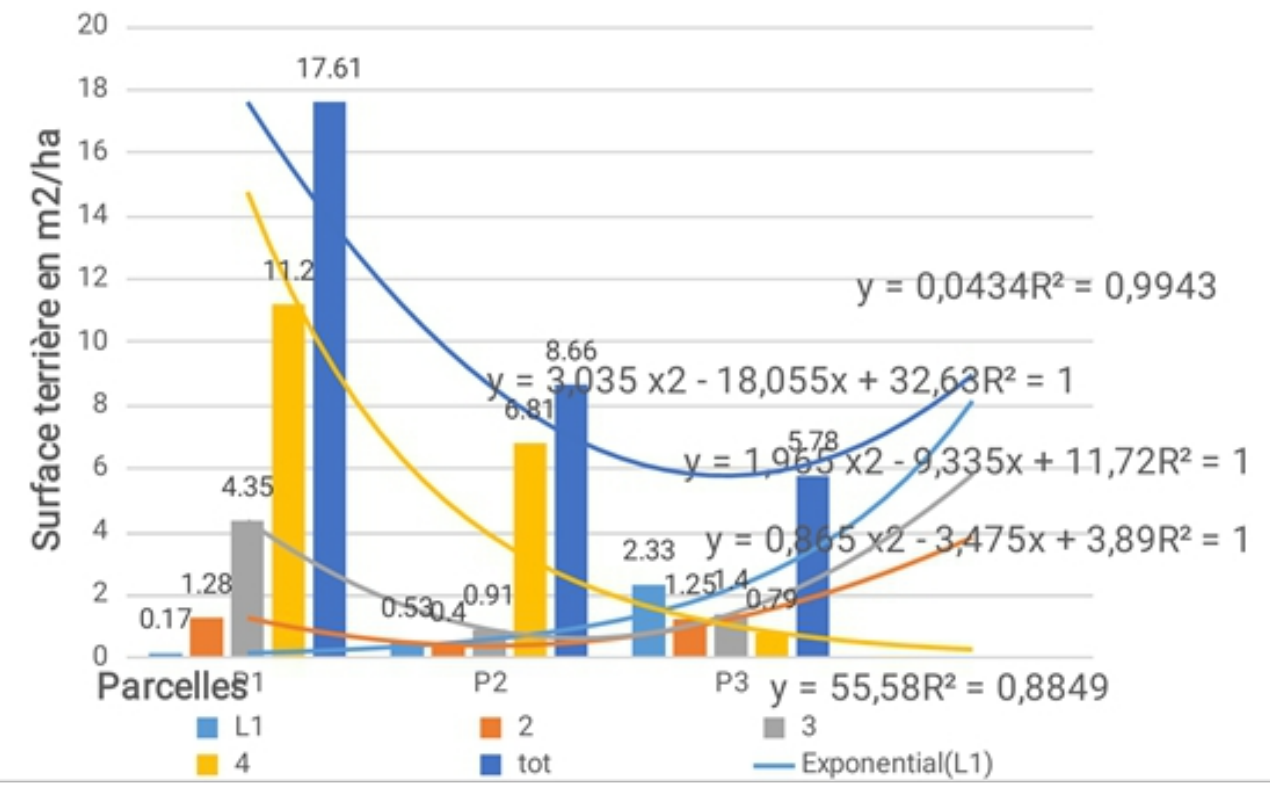

Figure 5. Différentes valeurs des surfaces terrières au sein de chaque parcelle et entre les parcelles

\section{Dominance relative et densité relative}

\section{Au niveau des espèces dans les 3 parcelles}

La flore de la zone d'étude est caractérisée par 5 espèces indicatrices et écologiquement importantes inventoriées dans les forêts sur terre ferme : Cynometra sessiliflora (densité $=408$ individus ; densité relative $=50,25 \%$ avec une dominance relative de $75,43 \%$; surface terrière $=24,19 \mathrm{~m} 2 /$ ha et IVI=141,34), Acacia siberiana (densité=106 individus, densité relative $=13,05 \%$, surface terrière $=2,48 \mathrm{~m} 2 / \mathrm{ha} ; \mathrm{DOR}=7,73 \%$ et IVI $=35,24)$,

Cola griseiflora (densité $=113$ individus ; $\mathrm{DR}=13,92 \%$; $\mathrm{ST}=1,51 \mathrm{~m} 2 / \mathrm{ha}$; 
DOR=4,71\% et IVI=29,47), Arthrosamanea obliquifoliolata (densité $=74$ individus ; $\mathrm{DR}=9,11 \%$; $\mathrm{DOR}=7,59 \% ; \mathrm{ST}=2,43 \mathrm{~m} 2 /$ ha et $\mathrm{IVI}=27,54)$ et Coelocaryon preussii (densité $=31$ individus; $\mathrm{DR}=3,82 \% ; \mathrm{DOR}=1,19 \%$; $\mathrm{ST}=0,38 \mathrm{~m} 2 /$ ha et $\mathrm{IVI}=12,23$ ). Les autres espèces sont faiblement représentées (80 individus ; $\mathrm{ST}=1,08 \mathrm{~m} 2 / \mathrm{ha} ; \mathrm{DR}=9,85 \% ; \mathrm{DOR}=3,35 \%$ et $\mathrm{IVI}=54,18$ ) dans toutes les 3 parcelles des forêts sur terre ferme. (ANOVA : F-statistic= 0.03747 ; p-value $=0.8783$ ). Il existe une forte variabilité des valeurs de caractéristiques structurales entre les espèces dans les parcelles des forêts sur terre ferme.

Légende : $\mathrm{ST}=$ Surface terrière, $\mathrm{FR}=$ Fréquence relative, $\mathrm{IVI}=$ Indices des Valeurs d'importance des espèces, $\mathrm{DOR}=$ dominance relative et $\mathrm{DR}=$ densité relative (Tableau 2).

Tableau 2. Caractéristiques structurales au niveau des espèces dans les forêts sur terre ferme

\begin{tabular}{|c|c|c|c|c|c|c|c|}
\hline Espèces & Densité & ST & DR(\%) & $\operatorname{DOR}(\%)$ & $\operatorname{FR}(\%)$ & IVI & P-value \\
\hline Acacia siberiana & 106 & 2,48 & 13,05 & 7,73 & 14,46 & 35,24 & \\
\hline Arthrosamanea & & & & & & & \\
\hline obliquifoliolata (Dwild) & & & & & & & \\
\hline Gilbert \& Bout & & & & & & & \\
\hline Coelocaryon preussii WARB. & 74 & 2,43 & 9,11 & 7,59 & 10,84 & 27,54 & \\
\hline Cola griseiflora DE WILD & 31 & 0,38 & 3,82 & 1,19 & 7,23 & 12,23 & \\
\hline Cynometra sessiliflora & 113 & 1,51 & 13,92 & 4,71 & 10,84 & 29,47 & \\
\hline$H A R M S$ & 408 & 24,19 & 50,25 & 75,43 & 15,66 & 141,34 & \\
\hline Autres & 80 & 1,08 & 9,85 & 3,35 & 40,97 & 54,18 & \\
\hline Total & 812 & 32,07 & 100,00 & 100,00 & 100,00 & 300,01 & $\mathbf{0 . 8 7 8 3}$ \\
\hline
\end{tabular}

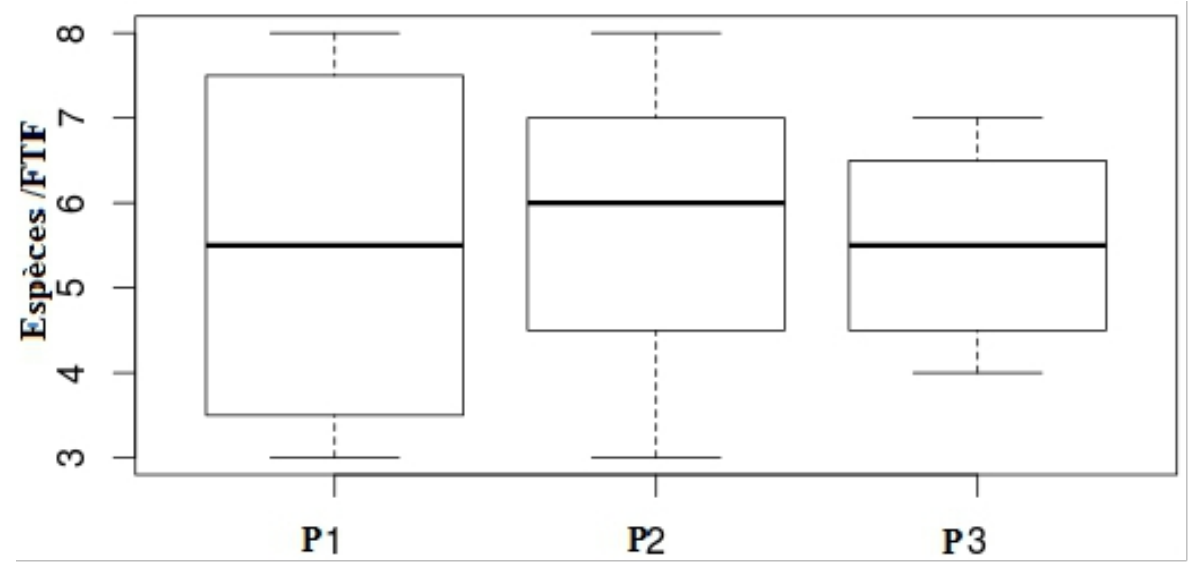

Figure 6. La boîte qui représente l'intervalle dans lequel sont groupés $50 \%$ d'observations des espèces dans les 3 parcelles des forêts sur terre ferme

\section{Indices des valeurs d'importance des Familles (FIV)}

La flore étudiée est dominée par 5 familles écologiquement importantes et les plus diversifiées, notamment les Familles des Fabaceae (DR=73,65\%, DOR=91,16\%, Densité $=598$ individus, ST=29,23m2/ha et $\mathrm{FIV}=179,1)$, les Malvaceae $\quad(\mathrm{DR}=16,01 \%$, Densité=130 individus, 
$\mathrm{DOR}=5,13 \%, \mathrm{ST}=1,64 \mathrm{~m} 2 / \mathrm{ha}$ et $\mathrm{FIV}=33,04)$, Combretaceae $(\mathrm{DR}=4,06 \%$, $\mathrm{DOR}=1,77 \%, \quad \mathrm{ST}=0,57 \mathrm{~m} 2 / \mathrm{ha}$, Densité $=33$ individus et $\mathrm{FIV}=15,36$ ), Myristicaceae (Densité $=31$ individus, $\mathrm{DR}=3,82 \%, \quad \mathrm{DOR}=1,19 \%$, $\mathrm{ST}=0,38 \mathrm{~m} 2 /$ ha et $\mathrm{FIV}=12,14)$ et les Ebenaceae $(\mathrm{DR}=3,82 \%$, DOR=0,34\%, Densité $=13$ individus, $\mathrm{ST}=0,11 \mathrm{~m} 2 / \mathrm{ha}$ et $\mathrm{FIV}=13,85)$. Au contraire, les autres familles enregistrent des faibles valeurs (Densité $=7$ espèces, $\mathrm{DR}=0,9 \%$, $\mathrm{DOR}=0,41 \%, \mathrm{ST}=0,07 \mathrm{~m} 2 / \mathrm{ha}$ et $\mathrm{FIV}=46,5)$. Il existe une forte variabilité entre les parcelles des forêts sur terre ferme.

Légende : $\mathrm{ST}=$ Surface terrière, $\mathrm{FR}=$ Fréquence relative, FIV=Indices des Valeurs d'importance des Familles, $\mathrm{DOR}=$ dominance relative et $\mathrm{DR}=$ densité relative et Diversité relative (Div.rel) (Tableau 3).

Tableau 3. Indices des valeurs d'importances des familles (FIV)

\begin{tabular}{|l|c|c|c|c|c|c|}
\hline Familles & Densité & ST & DOR(\%) & DR (\%) & $\begin{array}{c}\text { Div. } \\
\text { Rel(\%) }\end{array}$ & FIV \\
\hline Combretaceae & 33 & 0,57 & 1,77 & 4,06 & 9,52 & 15,36 \\
Ebenaceae & 13 & 0,11 & 0,34 & 1,60 & 11,90 & 13,85 \\
Fabaceae & 598 & 29,23 & 91,16 & 73,65 & 14,28 & 179,1 \\
Malvaceae & 130 & 1,64 & 5,13 & 16,01 & 11,90 & 33,04 \\
Myristicaceae & 31 & 0,38 & 1,19 & 3,82 & 7,14 & 12,15 \\
Autres & 7 & 0,07 & 0,41 & 0,9 & 45,24 & 46,5 \\
\hline Total & $\mathbf{8 1 2}$ & $\mathbf{3 2 , 0 7}$ & $\mathbf{1 0 0 , 0 1}$ & $\mathbf{1 0 0 , 0 0}$ & $\mathbf{1 0 0}$ & $\mathbf{3 0 0}$ \\
\hline
\end{tabular}

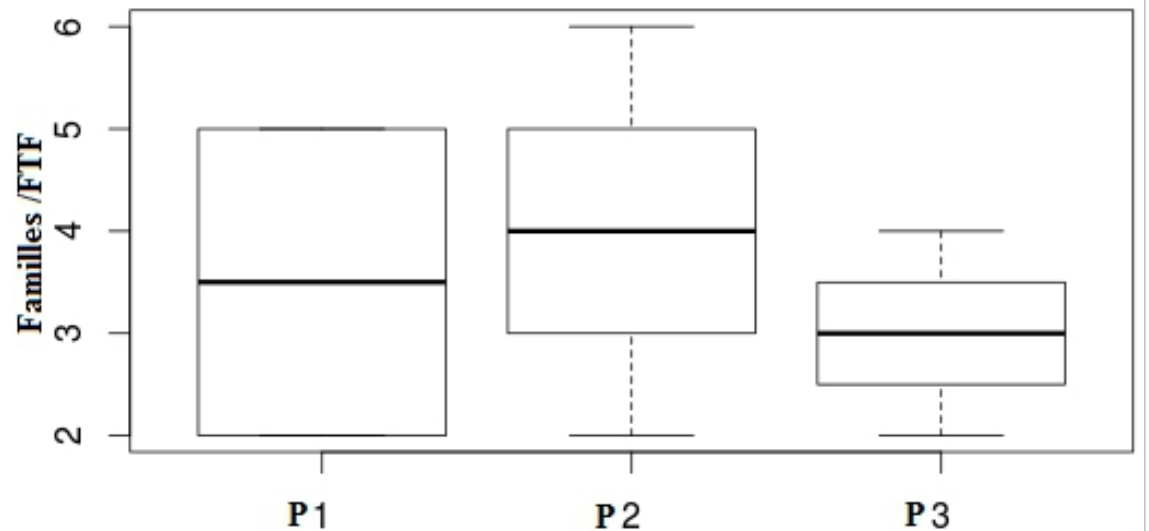

Figure 7. Représentation des 50\% d'observations groupés dans la boîte qui indique l'intervalle dans lequel les familles des forêts sur terre ferme sont représentées

\section{Structure Diamétrique}

La comparaison des structures diamétriques de tous les individus inventoriés à $\mathrm{Dhp} \geq 10 \mathrm{~cm}$ dans les forêts sur terre ferme montre un nombre très élevé pour les arbres dont les diamètres se situent entre les classes des diamètres de 10-20 cm (513 individus) où la parcelle $\mathrm{P} 1$ domine dans cette catégorie avec 204 arbres inventoriés. La faible valeur des diamètres est observée dans la parcelle P3 (136 arbres) et la classe de 40-50 $\mathrm{cm}$ de diamètre enregistre une faible valeur (19 arbres inventoriés). Le diamètre de l'arbre le 
plus gros dans les forêts sur terre ferme (Cynometra sessiliflora) est de 63,69 $\mathrm{cm}$, inventorié dans la parcelle P2. Il existe une forte variabilité des structures diamétriques des arbres inventoriés dans les 3 parcelles de ces forêts (ANOVA : F-statistic $=0.6941 ; \mathrm{p}$-value $=0.5578)$.

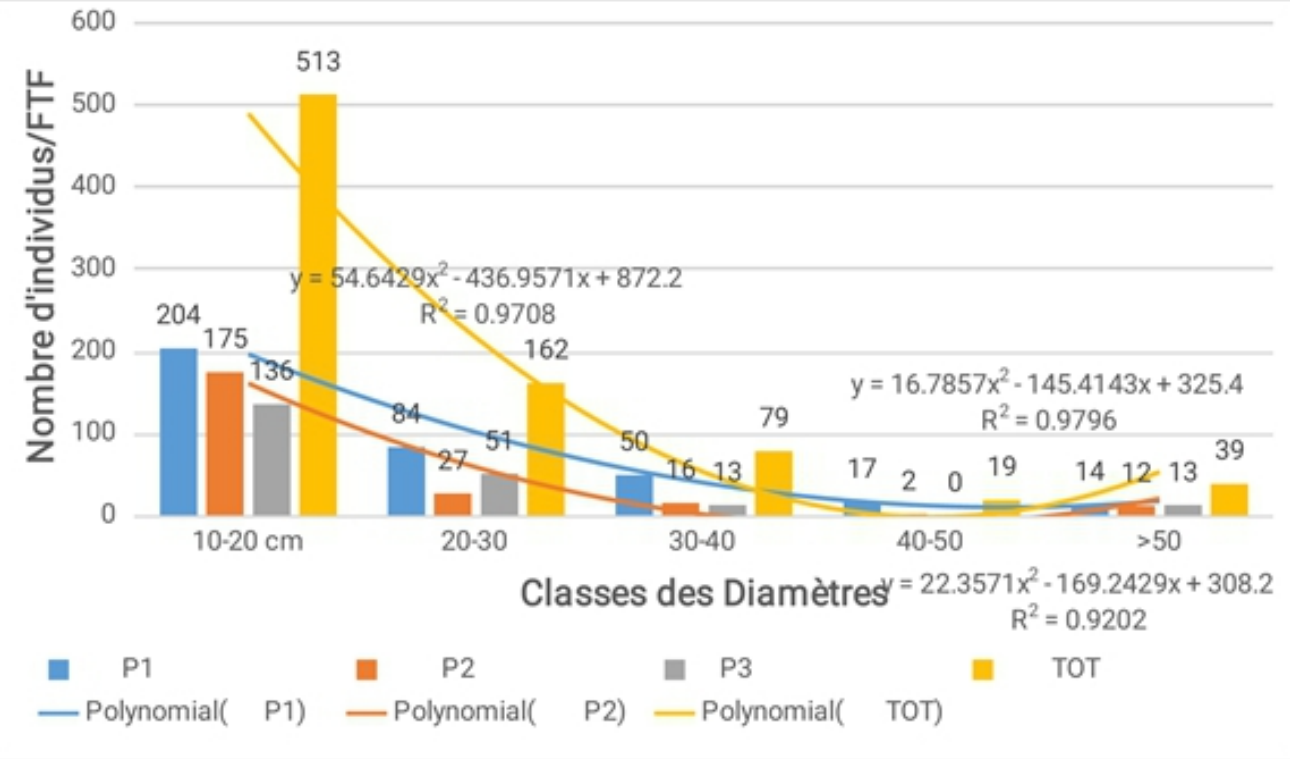

Figure 8. Distribution par classes diamétriques de tous les arbres inventoriés à $\mathrm{Dhp} \geq 10 \mathrm{~cm}$ dans les 3 parcelles

\section{Richesse et Indices de diversité}

\section{Diversité relative (Div. Rel.)}

La diversité relative des espèces au sein de ces 3 parcelles des forêts sur terre ferme varie d'une parcelle à l'autre. L'espèce Cynometra sessiliflora est écologiquement présente dans toutes les 3 parcelles (408 individus, soit $50,25 \%)$. Cette espèce est plus nombreuse dans la parcelle P1 avec 249 individus, soit $87,09 \%$; la parcelle P2 suit avec 105 individus soit 83,62\% et elle se présente faiblement dans la parcelle $\mathrm{P} 3$ avec 54 individus, soit 27,67\%. L'espèce Acacia siberiana est aussi présente dans toutes les 3 parcelles des forêts sur terre ferme (106 individus soit 13,05\%), se retrouvant plus dans la parcelle P3. En général, il y a une forte variabilité des espèces présentes dans les 3 parcelles de ces forêts. Certaines parcelles contiennent des espèces caractéristiques propres telles que: Acacia farnesiana (P3), Diospyros ituriensis (P1) et Vitex welwistchii (P1). 
Légende : Div. Rel = Diversité relative, $\%=$ Pourcentage.

Tableau 4. Diversité relative dans les 3 parcelles des forêts

\begin{tabular}{|l|c|c|}
\hline Espèces & Densité & Div. Rel (\%) \\
\hline Acacia farnesiana (L) WILD & 7 & 0,86 \\
Acacia siberiana & 106 & 13,05 \\
Arthrosamanea obliquifoliolata (Dwild) Gilbert \& Bout & 74 & \\
Caloncoba crepiniana (DE WILD \& TH.DUR) GILG & 3 & 9,11 \\
Coelocaryon preussii WARB. & 31 & \\
Cola congolana DE WILD \& TH.DUR & 15 & 0,37 \\
Cola griseiflora DE WILD & 113 & 3,82 \\
Combretum lokele LIBEN & 33 & 1,85 \\
Cynometra sessiliflora HARMS & 408 & 13,92 \\
Cynometra sessiliflora HARMS Var. laurentii (DE WILD) & & 4,06 \\
LEBRUN & & 50,25 \\
Diospiros polystemon GURKE & 3 & 0,37 \\
Diospyros ituriensis (GURKE) R.LET. \& F.WHITE & 12 & 1,48 \\
Ficus mucuso WELW.EX.FICALHO & 1 & 0,12 \\
Grewia seretii DE WILD & 1 & 0,12 \\
Vitex doniana Sweet & 2 & 0,25 \\
Vitex welwistchii GURKE & 2 & 0,25 \\
& 1 & 0,12 \\
\hline Total & $\mathbf{8 1 2}$ & $\mathbf{1 0 0}$ \\
\hline
\end{tabular}

\section{Richesse floristique}

L'inventaire réalisé dans les forêts sur terre ferme (3 Hectares) a donné 812 individus inventoriés sur une surface terrière totale de $32,06 \mathrm{~m}^{2} / \mathrm{ha}$, répartis dans 16 espèces, 11 genres et 8 familles.

La richesse spécifique varie d'une parcelle à l'autre dans les forêts sur terre ferme. L'Indice alpha de Fisher $\alpha$ calculé à Bootstrap (95\% confidence) in Past est respectivement de $(0,39 ; 0,87 ; 0,93 ; 0,87)$. Les valeurs de l'indice alpha de Fisher $\alpha$ varient d'une parcelle à l'autre. La variation de la richesse spécifique entre les 3 parcelles de ces forêts est remarquable (Le coefficient de variation $(C V)=29,6 \%$, Moyenne $=16,8$ et l'Écart type $=4,95)$.

Tableau 5. Richesses floristiques entre les parcelles

\begin{tabular}{|l|c|c|c|c|c|c|c|}
\hline Caractéristiques floristiques & P1 & P2 & P3 & Moyenne & Écart type & CV (\%) & Alpha Fisher \\
\hline Nombre d'individus & 384 & 233 & 195 & 203 & 22,1 & 10,9 & 0,39 \\
Richesse spécifique & 10 & 9 & 11 & 16,5 & 4,95 & 29,6 & 0,87 \\
Richesse générique & 9 & 7 & 9 & 15,3 & 3,89 & 25,5 & 0,93 \\
Richesse Familiale & 7 & 6 & 6 & 10,5 & 3,61 & 0,34 & 0,87 \\
\hline
\end{tabular}

\section{Indices de Similarité}

\section{Indice de Jaccard}

Etant donné que la valeur de l'indice de Jaccard est petite, la similarité est aussi faible dans la composition floristique entre les parcelles des forêts en étude. 
Tableau 6. Indices de Similarité de Jaccard

\begin{tabular}{|c|c|c|c|}
\hline Indice de Jaccard & P1 & P2 & P3 \\
\hline Parcelles & 1 & 1 & 1 \\
P1 & 1 & 1 & 1 \\
P2 & 1 & 1 & 1 \\
P3 &
\end{tabular}

\section{Indice de Morisita (MH)}

Une forte similitude est observée entre les parcelles de ces forêts.

Tableau 7. Les valeurs de l'indice de Morisita dans les forêts sur terre ferme

\begin{tabular}{|c|c|c|c|}
\hline \multicolumn{3}{|c|}{ Indice de Morisita } & P2 \\
\hline Parcelles & P1 & 0.9978 & P3 \\
P1 & 1 & 1 & 0.9999 \\
P2 & 0.9978 & 0.9988 & 0.9988 \\
P3 & 0.9999 & 1 \\
\hline
\end{tabular}

La variabilité floristique testée à partir de l'indice de Morisita dans cette étude a fait observer une forte similarité entre les parcelles alors qu'elles sont assez distantes géographiquement. Elles ont plus des relations floristiques les unes avec les autres.

\section{Discussion}

\section{Densité et Surface Terriere}

En comparant la densité dans chaque parcelle, il ressort que la parcelle (P1) est supérieure avec 384 individus inventoriés (47,29 \%), la parcelle ( P2) contient plus d'espèces que les autres parcelles $(84,61 \%)$, les parcelles (P1) et (P2) contiennent respectivement 9 et 5 genres chacune, la parcelle (P1) à la tête du nombre des familles, soit 7 (100\%), le diamètre supérieur d'un arbre est observé dans la parcelle (P2) soit 63,69 cm tandis que la surface terrière supérieure est obtenue dans la parcelle (P1) soit 17,61 m2/ha.. Ceci montre une forte variabilité de la densité spécifique dans ces forêts.

Lisingo et al. (2015) montrent «que les peuplements d'arbres présentent des variations notables dans leurs diversités spécifiques et leur composition floristique ; les sites septentrionaux de l'Ituri et de Rubi-Télé sont localement moins diversifiés que ceux de Yoko et de Uma aux environs de Kisangani|». Les forêts de l'Ile Mbiye (Nshimba, 2008), de Uma (Katembo, 2013), de Masako (Loris, 2009), de l'Uélé (Gerard, 1960) et de Yoko (Masiala, 2009) présentent une forte densité spécifique. Cependant, les faibles densités observées dans notre milieu d'étude sont liées à la présence des Talwegs en forêts et aux perturbations anthropiques.

Lomba (2012) a trouvé une valeur plus élevée de la surface terrière totale de $11.97 \mathrm{~m}^{2} /$ ha dans le dispositif des forêts à Yoko que dans les forêts de Biaro (RDC), avec une surface terrière totale de $9.75 \mathrm{~m}^{2} / \mathrm{ha}$. Il a justifié cette situation par la plus forte abondance d'individus de gros diamètre à Yoko 
par rapport aux diamètres des arbres observés à Biaro. Nos résultats ne sont pas loin de l'idée de Richards (1999) et Kouob (2009) qui stipulent que « dans les forêts tropicales, les densités des arbres d'au moins $10 \mathrm{~cm}$ de Dbh varient entre 300 et 700 arbres/ha ». Milenge (2013) fait observer que la composition floristique varie en fonction de la nature du substrat (sol inondable et terre ferme) et elle est déterminée à la fois par la texture et la composition chimique du sol.

\section{Dominance Relative et Densité Relative}

Cinq (5) espèces caractéristiques des forêts sur terre ferme dominent dans le milieu d'étude, dont l'espèce Cynometra sessiliflora est la plus importante dans toutes les 3 parcelles étudiées, respectivement (P1) avec $87,09 \%$, P2 avec 83,62\% et P3 avec 27,67 \%. Elle présente une densité spécifique supérieure de 408 individus $(50,25 \%)$ tandis que le reste des espèces varient d'une parcelle à l'autre.

Comparaison des valeurs d'importances des familles des différents sites étudiés par d'autres Auteurs

Légende : $\%$ = pourcentage, $1=$ Katembo Eric (2013), 2 = Katembo John (2013), 3 = Milenge (2013), 4 = Boyemba (2011), 5 = Mbayo (2017), $6=$ Nshimba (2008), 7 = Kangueja (2009), 8 = Somue (2009), $9=$ Lisingo (2009), $10=$ Mposo (2018).

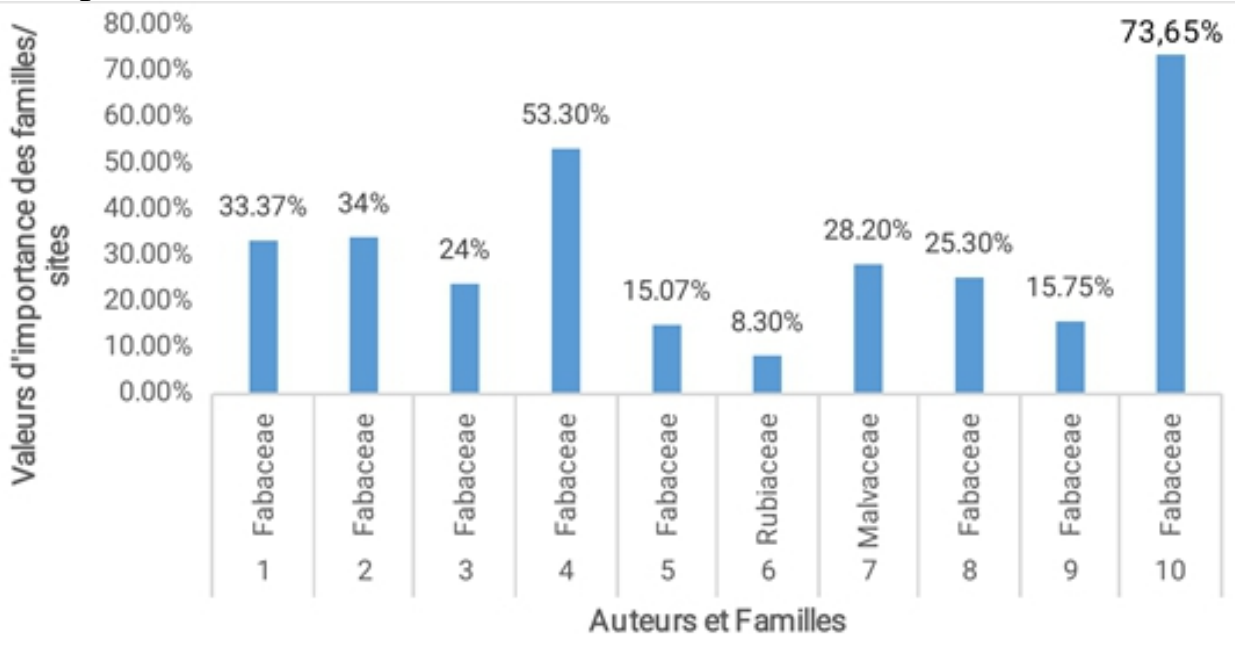

Figure 9. Comparaison des valeurs d'importance des familles dans les différents sites

\section{Structure Diamétrique}

Nos résultats montrent que les individus ayant les structures diamétriques se situant entre la classe des diamètres de 10-20 cm sont très 
nombreux (513 individus), suivis de la classe de 20-30 cm de diamètre avec 162 individus.

Cette différence de diamètres des arbres entre les parcelles s'explique par la présence d'un gradient des conditions écologiques plus favorables pour ces forêts où l'espèce Cynometra sessiliflora est dominante ; elle présente plus de tiges de $\mathrm{dhp} \geq 30 \mathrm{~cm}$. Ceci démontre que nombreux d'arbres inventoriés du lieu d'étude présentent des faibles diamètres car ils sont de petite taille. Bedel et al. (1998) ont observé que la structure diamétrique varie selon l'âge des forêts. Dupuy (1998) précise également que le peuplement forestier des forêts denses tropicales présente une structure décroissante où le nombre de tiges diminue au fur et à mesure que le diamètre augmente. Kangueja (2009) fait observer que le nombre d'individus de petits diamètres restent élevés. Boyemba (2011) fait observer dans le site de Yangambi que l'effectif est important (soit $62 \%$ de l'ensemble de tiges $\geq 10 \mathrm{~cm}$ de $\mathrm{dbp}$ ) pour la classe 10 $20 \mathrm{~cm}$ et ensuite une forte baisse des effectifs jusqu'à la classe $40-50 \mathrm{~cm}$. Nos résultats ne s'éloignent pas des observations faites par les autres auteurs précités.

\section{Richesse Specifique et Indices de Similarité}

Tenant compte des parcelles des forêts sur terre ferme en étude, un total de 812 individus (3 Hectares) ont été répartis dans 16 espèces, 11 genres et 8 familles inventoriés sur une moyenne de surface terrière totale de 32,06 $\mathrm{m}^{2} /$ ha. Katembo (2013) fait remarquer que les faibles valeurs des indices de diversité de la forêt d'Uma sont dues aux phénomènes de dominance relative entre les parcelles étudiées et il y a aussi une variabilité spécifique remarquable dans chaque parcelle. Ce constat ne s'éloigne pas de nos observations faites dans le milieu d'étude, selon lesquelles cinq espèces sur l'ensemble des espèces inventoriées dominent ces forêts.

La variabilité floristique testée à partir de l'indice de Morisita dans cette étude a fait observer une forte similarité entre les parcelles alors qu'elles sont assez distantes géographiquement. Elles ont plus des relations floristiques les unes avec les autres. Katembo (2013) fait remarquer que la composition spécifique est différente entre les parcelles. Cette différence peut être due à la distance géographique entre elles. Les mêmes observations ont été faites par Lisingo (2009) et Amani (2011) dans la réserve forestière de Yoko(RDC). La plus forte similarité observée entre les parcelles du même type forestier dans cette étude étant distantes les unes des autres se justifie parce qu'elles présentent presqu'une même composition floristique homogène influencée par les facteurs écologiques, composition où l'espèce de Cynometra sessiliflora qui domine avec 408 individus $(50,25 \%)$ se retrouve dans toutes les parcelles du milieu d'étude. (Lisingo, 2015) argumente que les forêts proches du Rift 
Albertin dans le Ruwenzori supposent des refuges forestiers à la lisière des forêts de l'Est de l'Afrique tropicale et celles proches du Mont Cameroun.

\section{Conclusion}

L'objectif de cette étude est d'analyser la variabilité structurale et floristique entre les parcelles des forêts sur terre ferme en chefferie de Bahema-Boga dans la province de l'Ituri en République Démocratique du Congo (RDC). Tenant compte des paramètres structuraux et floristiques des forêts, cette étude a permis d'observer les variabilités notables des structures et de la composition floristique au sein d'une même parcelle (hectare), entre les parcelles proches et celles distantes d'une même forêt sur terre ferme.

Les indices analysés ont fait observer une forte similarité entre les parcelles du même type forestier alors qu'elles sont assez distantes géographiquement. Elles ont plus de relations floristiques les unes avec les autres. Nous pouvons tirer des conclusions selon lesquelles les structurales et la composition floristique varient d'une parcelle à l'autre et d'une forêt à l'autre suite à l'influence des facteurs écologiques (texture du sol, type des peuplements). Milenge (2013) a trouvé que la composition floristique varie en fonction de la nature du substrat (sol inondable et terre ferme) et elle est déterminée à la fois par la texture et la composition chimique du sol.

La présente étude constitue donc une réponse positive à l'insuffisance des recherches scientifiques en écologie forestière dans la région du Bassin du Nil, particulièrement en chefferie de Bahema-Boga, prouvée par le manque d'aménagement des forêts dans cette région de l'Est de la République Démocratique du Congo.

Nos résultats peuvent renseigner à ceux qui sont intéressés par la gestion de l'environnement sur l'estimation de la quantité des émissions de $\mathrm{CO}_{2}$ séquestrée par la plante et estimer cette quantité de $\mathrm{CO} 2$ à l'hectare de ces forêts. Enfin, les résultats de la présente recherche en chefferie de Bahema -Boga sont également utiles pour tout scientifique qui voudra s'en servir pour se faire l'idée sur la structure et la composition floristique des forêts de la région d'étude dans le bassin du Nil à l'Est de la RDC pour permettre ainsi de proposer un plan de gestion rationnelle et d'aménagement durable des ressources forestières de la région.

\section{References:}

1. Apani \& Marcel Motom (2013). Analyse Phytoécologique De La Flore Ligneuse De La Haute Sangha (République Du Congo) École Normale Supérieure, Université Marien Ngouabi, Boîte Postale 237, Brazzaville (République Du Congo) 134p. 
2. Boyemba, B. (2006). Diversité Et Régénération Des Essences Forestières Exploitées Dans Les Forêts Des Environs De Kisangani (Rd Congo). Mémoire De Dea, Ulb, Bruxelles, $101 \mathrm{P}$.

3. Boyemba, F. B. (2011). Ecologie De Pericopsis Elata (Harms) Van Meeuwen (Fabaceae), Arbre De Forêt Tropicale Africaine A Répartition Agrégée. Thèse De Doctorat, Université Libre De Bruxelles, Belgique, $181 \mathrm{P}$.

4. Braun-Blanquet, J. (1932). Plant Sociology: The Study Of Plant Communities. Mc Graw-Hill Book Company, Inc., New York, 407 P.

5. Chave, J. (2000). Dynamique Spatio-Temporelle De La Forêt Tropicale. Ann. Phys. Fr. $25 . \mathrm{N}^{\circ} 6$ :1-184.P

6. Clark, D.B. \& Clark, D.A. (2000). Lanscape-Scale Variation In Forest Structure Biomass In A Tropical Rain Forest. Forest Ecology And Management, 137: 185-198.

7. Clements, F. E. (1916). Plant Succession: Analysis of Development of Vegetation. Publs. Carnegie Instn. 242, Washington, D. C.

8. Defourny, P. \& Eba'a, R. (Eds.) (2012). Les Forêts Du Bassin Du Congo - Etat Des Forêts 2010, Luxembourg : Office Des Publications De L'union Européenne, 276 P.

9. Fournier, F. \& Sasson, A. (1983). Les Ecosystèmes Forestiers Tropicaux D'afrique, Paris, $473 \mathrm{P}$

10. Gérard, R. (1960). Etude Écologique De La Forêt À Gilbertiodendron Dewevrei Dans La Région de L'uéle. Publ. Ineac, Série Scient. $\mathrm{N}^{\mathrm{O}}$ 87, Bruxelles, $159 \mathrm{P}$.

11. Gillet, F. (2000). La Phytosociologie Synusiale Intégrée: Guide Méthodologique. Laboratoire D'écologie Végétale Et De Phytosociologie De L'université De Neuchatel, Inst. De Bot.: 65 P.

12. Gounot, M. (1969). Méthode Quantitative De La Végétation. Ed. Masson Et Cie, Paris, 314p.

13. Harvey, J.M. (2002). Biostatistique. Une approche intuitive. De Boeck Université, p257-288.

Http://Carpe.Umd.Edu/Products/Pdf_Files/Focb_Aprelimassess.Pdf $39 \mathrm{p}$.

14. Kangueja Bukasa (2010). Analyse De La Diversité Des Ligneux Arborescents Des Principaux Types Forestiers Du Nord-Est De La Réserve De Biosphère De Luki (Bas-Congo, (R.D.C), Mémoire, Université De Kisangani, 198p.

15. Katembo Mukirania (2013). Etude De La Variabilité Structurale Et Floristique Dans Les Forêt De Uma,Rdcono, Mémoire De Des/Dea F.S/Unikis, 63p. 
16. Kouob, B. S. (2009). Organisation De La Diversité Végétale Dans Les Forêts Matures De Terre Ferme Du Sud-Est Cameroun. Thèse Université Libre De Bruxelles, 188 P.

17. Lebrun, J. \& Gilbert, G. (1954). Une Classification Écologique Des Forêts Du Congo. Bruxelles, Belgique, I.N.E.A.C., 63, 89 P.

18. Lejoly, J., Ndjele, M-B. \& Geerinck, D. (2010). Catalogue-Flore Des Plantes VasculaireDes Districts De Kisangani Et De La Tshopo (Rd Congo). Taxonomania 30: 1-308. (Bruxelles).

19. Lisingo, W.L. (2009). Typologie Des Forêts Denses Des Environs De Kisangani Par Une Méthode D'analyse Phytosociologique Multistrate, Dea, Faculté Des Sciences, Unikis. 91 P.

20. Lomba, B. \& Ndjele, M. B. (1998). Utilisation De La Méthode De Transect En Vue De L'étude De La Phytodiversité Dans La Réserve De Yoko, Ubundu, République Démocratique Du Congo. Annales Faculté Des Sciences, 11, Unikis, 35 - 45 P.

21. Louis, J. (1947). Contribution à l'étude des forêts équatoriales congolaises. C.R. Sem. Agr. INEAC Yangambi. 902-924p

22. Magurran, A.E. (2004). Measuring Biological Diversity. Blackwell Publishing Company, United Kingdom. 256p.

23. Makana, J. R. (2004). Ecology And Sustainable Management Of African

24. Mohaganies And Other Selected Timber Species In Northeastern Congo Basin, Democratic Republic Of Congo. Thesis For Degree Of Doctor, University Of Toronto, Usa, $215 \mathrm{P}$.

25. Mandango, M. A. (1982). Flore Et Végétation Des Iles Du Fleuve Zaïre Dans La Sous Région De La Tshopo (Haut-Zaïre). Thèse De Doctorat, Université De Kisangani. Tome 1, 425p.

26. Masiala, G. (2009). Analyse d'une zone de contact de la forêt à Gilbertiodendon dewevrei (De Wild.)J. Léonard avec la forêt semi-caducifoliée dans la réserve de la Yoko nord (RDC). Mémoire inédit de D.E.S. Faculté de sciences, Université de Kisangani. 103p.

27. Mbayo, M. F. (2017). Foret à maranthaceae et son impact sur la regenerations des ligneux dans la reserve forestiere de Yoko (Ubundu, Province de la Tshopo, R.D.Congo), These de Doctorat en sciences Agronomiques, faculte des Gestions des ressources Naturelles renouvelables (FGRNR), Universite de Kisangani, 249P.

28. Milenge Kamalebo Héritier (2013). Facteur Déterminant L'organisation Spatiale Et La Diversité Floristique Des Strates Arborescentes Des Forêts De L'ile Kongolo A Kisangani/R.D. Congo, Mémoire (D.E.S/D.E.A),I59p. 
29. Ministere De L'environnement Rapport Intermédiaire Sur La Mise En Euvre De La Convention Relative A La Biodiversité En République Démocratique Du Congo (1997). 101p.

30. Ndovya Gloire (2013). Estimation Automatisée Par Lidar Terrestre Comparé Au Laser Ace Et Vertex Des Paramètres Dendrométriques Des Arbres A Défaut De Conformation En Forêt Naturelle Dense Humide: Cas De La Forêt De Biaro (Kisangani, R.D.Congo) 39p.

31. Nebel, G., Kvist, L. P., Vanclay, J. K., Christensen, H. \& Freistas, L. (2001). Structure and floristic composition of flood plain forests in the Peruvian Amazon: I. Overstorey. Forest Ecology and Management 150 (1-2): 27-57

32. Nshimba, H. (2005). Etude floristique, écologique et phytosociologique des forêts inondées de l'île Mbiye à Kisangani, (RDC), DEA, ULB, $101 \mathrm{p}$.

33. Nshimba, H. (2008). Etude Floristique, Ecologique Et Phytosociologique Des Forêts De L'île Mbiye A Kisangani, R. D. Congo. Thèse, Université Libre De Bruxelles, 272 P.

34. Paget, D. (1999). Etude de la diversité spatiale des écosystèmes forestiers guyanais : réflexion méthodologique et application. Thèse de Doctorat, ENGREF, Paris. 154 pp.

35. Pascal, J.P. (2003). Notions sur les structures et dynamique des forêts tropicales humides. Rév. For. Fr. LV. Numéro spécial.

36. Pelissier, R., Couteron, P. \& Hardy, O. (2010). Organisation spatiale de la diversité des arbres des forêts tropicales aux échelles régionales : enjeux méthodologiques et application. Tropical forest ecology, IRD.p 149-163.

37. Richards, P.W. (1952). The rain forest, an ecological study, Cambridge University Press, $450 \mathrm{p}$.

38. Senterre, B. (2005). Recherches Méthodologiques Pour La Typologie De La Végétation Et La Phytogéographie Des Forêts Denses D'afrique Tropi. 